\title{
A TABLE OF ELLIPTIC INTEGRALS: ONE QUADRATIC FACTOR
}

\author{
B. C. CARLSON
}

\begin{abstract}
Integration in terms of real quantities is accomplished for 33 integrands that are rational except for the square root of a cubic or quartic polynomial with exactly one pair of conjugate complex zeros. Formulas are provided by which 45 more integrals of the same type can be expressed in terms of real quantities with the help of earlier papers. Neither limit of integration is assumed to be a singular point of the integrand. All the integrals are reduced to $R$-functions, for which Fortran programs are available. Most of the integrals are not listed in other tables.
\end{abstract}

\section{INTRODUCTION}

This paper treats integrands that are rational except for the square root of a cubic or quartic polynomial with exactly one pair of conjugate complex zeros. References $[4,5]$ dealt with elliptic integrals of the form

$$
[p]=\left[p_{1}, \ldots, p_{5}\right]=\int_{y}^{x} \prod_{i=1}^{5}\left(a_{i}+b_{i} t\right)^{p_{i} / 2} d t,
$$

where all quantities are real, $p_{1}, \ldots, p_{5}$ are integers (omitted if 0 ), and the number of odd $p$ 's is exactly three ("cubic cases") or four ("quartic cases"). Quartic cases were reduced by recurrence relations to the integrals

$$
\begin{array}{ll}
I_{1}=[-1,-1,-1,-1], & I_{2}=[1,-1,-1,-3], \\
I_{3}=[1,-1,-1,-1,-2], & I_{3}^{\prime}=[1,-1,-1,-1],
\end{array}
$$

and cubic cases were reduced to

$$
I_{1 c}=[-1,-1,-1], \quad I_{2 c}=[1,-1,-1], \quad I_{3 c}=[1,-1,-1,-2] .
$$

All seven of these integrals have $p_{2}=p_{3}$.

In $\S \S 2$ and 3 we consider integrals in which $p_{2}=p_{3}$ but $a_{2}+b_{2} t$ and $a_{3}+b_{3} t$ are conjugate complex:

$$
\left[p_{1}, p_{2}, p_{2}, p_{4}, p_{5}\right]=\int_{y}^{x}\left(f+g t+h t^{2}\right)^{p_{2} / 2} \prod_{i=1,4,5}\left(a_{i}+b_{i} t\right)^{p_{1} / 2} d t,
$$

Received December 7, 1989.

1980 Mathematics Subject Classification (1985 Revision). Primary 33A25; Secondary 33A30.

This work was supported by the Director of Energy Research, Office of Basic Energy Sciences. The Ames Laboratory is operated for the U.S. Department of Energy by Iowa State University under contract W-7405-ENG-82. 
where all quantities are real, $x>y, f+g t+h t^{2}>0$ for all real $t, p_{1}$ and $p_{2}$ are odd integers, $p_{4}$ may be odd or even, and $p_{5}$ is even (zero if $p_{4}$ is even). Section 2 contains quartic cases $\left(p_{4}\right.$ odd) and $\S 3$ contains cubic cases $\left(p_{4}\right.$ even). Proofs are given in $\S \S 4$ and 5 . All integral formulas have been checked by numerical integration; some details of the checks are given in $\S 6$.

We assume that the integral is well defined, possibly as a Cauchy principal value, and, in particular, that $a_{i}+b_{i} t>0$ for $y<t<x$ if $p_{i}$ is odd. The formulas of $[4,5]$ still hold but contain $a_{2}, b_{2}$, and their complex conjugates. The goal is to rewrite these formulas in terms of real quantities.

The integrals $I_{1}, I_{2}, \ldots, I_{3 c}$ are expressed in terms of four $R$-functions:

$$
\begin{gathered}
R_{F}(x, y, z)=\frac{1}{2} \int_{0}^{\infty}[(t+x)(t+y)(t+z)]^{-1 / 2} d t \\
R_{J}(x, y, z, w)=\frac{3}{2} \int_{0}^{\infty}[(t+x)(t+y)(t+z)]^{-1 / 2}(t+w)^{-1} d t
\end{gathered}
$$

and two special cases,

$$
R_{C}(x, y)=R_{F}(x, y, y) \text { and } R_{D}(x, y, z)=R_{J}(x, y, z, z) .
$$

When their last argument is negative, $R_{C}$ and $R_{J}$ are interpreted as Cauchy principal values. The functions $R_{F}, R_{D}$, and $R_{J}$ respectively replace Legendre's elliptic integrals of the first, second, and third kinds, while $R_{C}$ includes the inverse circular and inverse hyperbolic functions. Fortran codes for numerical computation of all four functions, including Cauchy principal values, are listed in the Supplements to $[3,4]$.

The main task is to express $I_{1}, I_{2}, \ldots, I_{3 c}$ in terms of real quantities by using Landen's transformation of $R_{F}$ and $R_{J}$. It is then simple to put formulas from $[4,5]$ with $p_{2}=p_{3}$ in real form. However, cases like $[1,-1,-1,1]$ require further work with recurrence relations because they are not listed in $[4$, $5]$, where the odd $p$ 's are always in descending order.

\section{TABLE OF QUARTIC CASES}

All quartic cases are reduced to the integrals $I_{1}, I_{2}, I_{3}$, and $I_{3}^{\prime}$. We assume $x>y, a_{1}+b_{1} t>0$ and $a_{4}+b_{4} t>0$ for $y<t<x$, and $f+g t+h t^{2}>0$ for all real $t$. The first set of definitions will apply in $\S 3$ to cubic cases also:

$$
\begin{gathered}
X_{i}=\left(a_{i}+b_{i} x\right)^{1 / 2}, \quad Y_{i}=\left(a_{i}+b_{i} y\right)^{1 / 2}, \quad d_{i j}=a_{i} b_{j}-a_{j} b_{i}, \\
\alpha_{i}=2 f b_{i}-g a_{i}, \quad \beta_{i}=g b_{i}-2 h a_{i}, \quad \delta^{2}=4 f h-g^{2}>0, \\
c_{i j}^{2}=2 f b_{i} b_{j}-g\left(a_{i} b_{j}+a_{j} b_{i}\right)+2 h a_{i} a_{j}, \\
\xi=\left(f+g x+h x^{2}\right)^{1 / 2}, \quad \eta=\left(f+g y+h y^{2}\right)^{1 / 2}, \\
A\left(p_{1}, p_{2}, p_{2}, p_{4}, p_{5}\right)=X_{1}^{p_{1}} \xi^{p_{2}} X_{4}^{p_{4}} X_{5}^{p_{5}}-Y_{1}^{p_{1}} \eta^{p_{2}} Y_{4}^{p_{4}} Y_{5}^{p_{5}} .
\end{gathered}
$$


The following definitions are used only in quartic cases:

$$
\begin{gathered}
(x-y) M=\left(X_{1} Y_{4}+Y_{1} X_{4}\right)\left[(\xi+\eta)^{2}-h(x-y)^{2}\right]^{1 / 2} \\
=\left(X_{1} Y_{4}+Y_{1} X_{4}\right)[2 \xi \eta+2 f+g(x+y)+2 h x y]^{1 / 2} \\
L_{ \pm}^{2}=M^{2}+c_{14}^{2} \pm c_{11} c_{44}, \quad W_{+}^{2}=M^{2}+d_{14}\left(c_{15}^{2}+c_{11} c_{55}\right) / d_{15} \\
(x-y) U=X_{1} X_{4} \eta+Y_{1} Y_{4} \xi, \quad W^{2}=U^{2}-c_{11}^{2} d_{45} / 2 d_{15} \\
Q=X_{5} Y_{5} W / X_{1} Y_{1}, \quad P^{2}=Q^{2}+c_{55}^{2} d_{45} / 2 d_{15} .
\end{gathered}
$$

When $a_{5}=1$ and $b_{5}=0$, the quantities $W^{2}, Q$, and $P^{2}$ reduce to

$$
W_{1}^{2}=U^{2}-c_{11}^{2} b_{4} / 2 b_{1}, \quad Q_{1}=W_{1} / X_{1} Y_{1}, \quad P_{1}^{2}=Q_{1}^{2}+h b_{4} / b_{1},
$$

and $W_{+}^{2}-M^{2}$ reduces to

$$
\rho=d_{14}\left[\beta_{1}-(2 h)^{1 / 2} c_{11}\right] / b_{1} .
$$

When one limit of integration is infinite, $M, U$, and $Q$ become, for $x=$ $+\infty$,

$$
\begin{aligned}
M & =\left(b_{1}^{1 / 2} Y_{4}+b_{4}^{1 / 2} Y_{1}\right)\left(2 h^{1 / 2} \eta+g+2 h y\right)^{1 / 2}, \\
U & =\left(b_{1} b_{4}\right)^{1 / 2} \eta+h^{1 / 2} Y_{1} Y_{4}, \quad Q=\left(b_{5} / b_{1}\right)^{1 / 2} Y_{5} W / Y_{1},
\end{aligned}
$$

or, for $y=-\infty$,

$$
\begin{aligned}
M & =\left[\left(-b_{1}\right)^{1 / 2} X_{4}+\left(-b_{4}\right)^{1 / 2} X_{1}\right]\left(2 h^{1 / 2} \xi-g-2 h x\right)^{1 / 2}, \\
U & =\left(b_{1} b_{4}\right)^{1 / 2} \xi+h^{1 / 2} X_{1} X_{4}, \quad Q=\left(b_{5} / b_{1}\right)^{1 / 2} X_{5} W / X_{1} .
\end{aligned}
$$

The four basic integrals (1.2) are

(2.14) $I_{1}=4 R_{F}\left(M^{2}, L_{-}^{2}, L_{+}^{2}\right)$,

$$
\begin{array}{r}
I_{2}=\left(2 c_{11} / 3 c_{44}\right)\left[4\left(c_{14}^{2}+c_{11} c_{44}\right) R_{D}\left(M^{2}, L_{-}^{2}, L_{+}^{2}\right)\right. \\
\left.-6 R_{F}\left(M^{2}, L_{-}^{2}, L_{+}^{2}\right)+3 / U\right]+2 X_{1} Y_{1} / X_{4} Y_{4} U \\
I_{3}=\left(2 c_{11} / 3 c_{55}\right)\left[4\left(d_{14} / d_{15}\right)\left(c_{15}^{2}+c_{11} c_{55}\right) R_{J}\left(M^{2}, L_{-}^{2}, L_{+}^{2}, W_{+}^{2}\right)\right. \\
\left.-6 R_{F}\left(M^{2}, L_{-}^{2}, L_{+}^{2}\right)+3 R_{C}\left(U^{2}, W^{2}\right)\right]
\end{array}
$$

$$
+2 R_{C}\left(P^{2}, Q^{2}\right)
$$

$$
I_{3}^{\prime}=\left(2 c_{11}^{2} / 9 h\right)^{1 / 2}\left[4 \rho R_{J}\left(M^{2}, L_{-}^{2}, L_{+}^{2}, M^{2}+\rho\right)-6 R_{F}\left(M^{2}, L_{-}^{2}, L_{+}^{2}\right)\right.
$$

$$
\left.+3 R_{C}\left(U^{2}, W_{1}^{2}\right)\right]
$$

$$
+2 R_{C}\left(P_{1}^{2}, Q_{1}^{2}\right)
$$


Integrals that converge when one limit of integration is infinite do not involve $I_{3}^{\prime}$.

Of the 41 quartic cases listed in [ $\left.{ }^{1}\right], 29$ have $p_{2}=p_{3}$. In these 29 cases the formulas given there can be used to evaluate

$$
\left[p_{1}, p_{2}, p_{2}, p_{4}, p_{5}\right]=\int_{y}^{x}\left(f+g t+h t^{2}\right)^{p_{2} / 2} \prod_{i=1,4,5}\left(a_{i}+b_{i} t\right)^{p_{i} / 2} d t
$$

by using (2.13) to (2.17) and usually doing a small amount of algebra to express coefficients involving $a_{2}, b_{2}, a_{3}, b_{3}$ in terms of $f, g$, and $h$. To aid in doing so, we list several identities:

$$
\begin{aligned}
& b_{2} b_{3}=h, \quad d_{2 i} d_{3 i}=h b_{i}^{2} r_{2 i} r_{3 i}=c_{i i}^{2} / 2, \quad d_{23}^{2}=h^{2} r_{23}^{2}=-\delta^{2}, \\
& a_{2} d_{3 i}+a_{3} d_{2 i}=\alpha_{i}, \quad b_{2} d_{3 i}+b_{3} d_{2 i}=\beta_{i}, \quad d_{2 i} d_{3 j}+d_{2 j} d_{3 i}=c_{i j}^{2}, \\
& r_{2 i}+r_{3 i}=\beta_{i} / h b_{i}, \quad r_{2 i}^{2}+r_{3 i}^{2}=\left(c_{i i}^{2} / h b_{i}^{2}\right)-\delta^{2} / h^{2}, \\
& r_{2 i}^{-1}+r_{3 i}^{-1}=2 b_{i} \beta_{i} / c_{i i}^{2}, \quad r_{i 2}-r_{3 j}=r_{i 2}+r_{j 3}=a_{i} / b_{i}+a_{j} / b_{j}-g / h .
\end{aligned}
$$

We recall that $d_{i j}$ and $r_{i j}=d_{i j} / b_{i} b_{j}$ are antisymmetric in $i$ and $j$.

Instead of rewriting all 29 formulas in terms of $f, g$, and $h$, it should suffice to give two examples. A simple one is

$$
\begin{aligned}
{[1,1,1,-3]=} & {\left[2\left(b_{1} \beta_{4}+h d_{14}\right) I_{3}^{\prime}+3 b_{1} c_{44}^{2} I_{2}-b_{4} c_{11}^{2} I_{1}\right] / 4 b_{1} b_{4}^{2} } \\
& +A(1,1,1,-1) / b_{4},
\end{aligned}
$$

and one of the most complicated is

$$
\begin{aligned}
{[1,1,1,-1,-4]=} & {\left[\left(\beta_{5} / b_{5}-c_{45}^{2} / d_{45}+b_{1} c_{55}^{2} / b_{5} d_{15}\right) I_{3}+4 h I_{3}^{\prime} / b_{5}\right.} \\
& \left.+c_{44}^{2} I_{2} / d_{45}-c_{11}^{2} I_{1} / d_{15}-4 A(1,1,1,-1,-2)\right] / 4 b_{5} .
\end{aligned}
$$

The coefficient of $I_{3}$ has been slightly simplified by using the identities

$$
2 h d_{i j}=b_{i} \beta_{j}-b_{j} \beta_{i}, \quad d_{i j} \beta_{k}=b_{i} c_{j k}^{2}-b_{j} c_{i k}^{2} .
$$

Among integrals with $p_{2}=p_{3}, \sum\left|p_{i}\right| \leq 8$, and $\sum p_{i} \leq 0$, there are 16 that are not listed in [4] because the odd $p$ 's are not in descending order. Six are integrals of the second kind with $p_{5}=0$ and $\sum p_{i}<-2$ :

$$
\begin{aligned}
{[-3,-1,-1,-3]=} & {\left[\left(b_{4}^{2}+b_{1}^{2} c_{44}^{2} / c_{11}^{2}\right) I_{2}-2 b_{1} b_{4} I_{1}\right] / d_{14}^{2} } \\
& +4 b_{1}^{2} A(-1,1,1,-1) / d_{14} c_{11}^{2},
\end{aligned}
$$

$$
\begin{gathered}
{[-3,1,1,-3]=\left(c_{44}^{2} I_{2}-c_{14}^{2} I_{1}\right) / d_{14}^{2}+2 A(-1,1,1,-1) / d_{14},} \\
{[-1,1,1,-5]=\left[-c_{14}^{2} I_{2}+c_{11}^{2} I_{1}-2 d_{14} A(1,1,1,-3)\right] / 3 d_{14}^{2},} \\
{[1,-3,-3,-1]=\left[\beta_{4} I_{2}-\beta_{1} I_{1}+2(g+2 h x) X_{1} / X_{4} \xi\right.} \\
\left.-2(g+2 h y) Y_{1} / Y_{4} \eta\right] / \delta^{2}
\end{gathered}
$$




$$
\begin{gathered}
{[1,-3,-3,1]=\left[-c_{44}^{2} I_{2}+c_{14}^{2} I_{1}-2\left(\alpha_{4}+\beta_{4} x\right) X_{1} / X_{4} \xi\right.} \\
\left.+2\left(\alpha_{4}+\beta_{4} y\right) Y_{1} / Y_{4} \eta\right] / \delta^{2} \\
{[-1,-3,-3,-1]=\left(2 / \delta^{2} c_{11}^{2}\right)\left\{\left(\delta^{2} b_{1} b_{4}-h c_{14}^{2}\right) I_{2}+h c_{11}^{2} I_{1}\right.} \\
+2\left[\delta^{2} b_{1}-h\left(\alpha_{1}+\beta_{1} x\right)\right] X_{1} / X_{4} \xi \\
\left.-2\left[\delta^{2} b_{1}-h\left(\alpha_{1}+\beta_{1} y\right)\right] Y_{1} / Y_{4} \eta\right\} .
\end{gathered}
$$

Four integrals of the third kind with $p_{5}=0$ or 2 and $\sum p_{i} \geq-2$ involve $I_{3}^{\prime}$ but not $I_{3}$ :

$$
\begin{aligned}
{[1,-1,-1,1]=} & {\left[\left(-b_{1} \beta_{4}-b_{4} \beta_{1}\right) I_{3}^{\prime}+b_{1} c_{44}^{2} I_{2}-b_{4} c_{11}^{2} I_{1}\right] / 4 h b_{1} } \\
& +b_{4} A(1,1,1,-1) / h
\end{aligned}
$$

$$
\begin{aligned}
{[-1,1,1,-3]=(} & \left.2 h d_{14} I_{3}^{\prime}+b_{1} c_{44}^{2} I_{2}-b_{4} c_{11}^{2} I_{1}\right) / 2 b_{1} b_{4} d_{14} \\
{[-1,1,1,-1]=[} & \left.\left(b_{1} \beta_{4}+b_{4} \beta_{1}\right) I_{3}^{\prime}+b_{1} c_{44}^{2} I_{2}+b_{4} c_{11}^{2} I_{1}\right] / 4 b_{1}^{2} b_{4} \\
& +A(1,1,1,-1) / b_{1}, \\
{[-1,1,1,-3,2]=} & {\left[\left(b_{1} b_{5} \beta_{4}+b_{4} b_{5} \beta_{1}-4 h b_{1} d_{45}\right) I_{3}^{\prime}\right.} \\
& +\left(b_{5}-2 b_{1} d_{45} / d_{14}\right) b_{1} c_{44}^{2} I_{2} \\
& \left.\quad+\left(b_{5}+2 b_{1} d_{45} / d_{14}\right) b_{4} c_{11}^{2} I_{1}\right] / 4 b_{1}^{2} b_{4}^{2} \\
& +b_{5} A(1,1,1,-1) / b_{1} b_{4} .
\end{aligned}
$$

In the last integral, $a_{5}$ and $b_{5}$ are unrestricted.

Six more quartic cases with $p_{5}=-2$ or -4 involve $I_{3}$, and the three with $\sum p_{i} \geq-2$ involve $I_{3}^{\prime}$ also:

$$
[1,-1,-1,1,-2]=\left(d_{45} I_{3}+b_{4} I_{3}^{\prime}\right) / b_{5},
$$

$$
\begin{aligned}
{[3,-1,-1,1,-2]=} & d_{15} d_{45} I_{3} / b_{5}^{2} \\
+ & {\left[\left(4 h b_{4} d_{15} / b_{5}-b_{1} \beta_{4}-b_{4} \beta_{1}\right) I_{3}^{\prime}\right.} \\
& +b_{1} c_{44}^{2} I_{2}-b_{4} c_{11}^{2} I_{1} \\
& \left.+4 b_{1} b_{4} A(1,1,1,-1)\right] / 4 h b_{5},
\end{aligned}
$$

(2.35) $[-1,1,1,-1,-2]=\left(b_{1} c_{55}^{2} I_{3}+2 h d_{15} I_{3}^{\prime}-b_{5} c_{11}^{2} I_{1}\right) / 2 b_{1} b_{5} d_{15}$,

(2.36) $[-1,1,1,-3,-2]=\left(d_{14} c_{55}^{2} I_{3}-d_{15} c_{44}^{2} I_{2}+d_{45} c_{11}^{2} I_{1}\right) / 2 d_{14} d_{15} d_{45}$,

$$
\begin{aligned}
{[1,-1,-1,1,-4]=} & {\left[\left(2 d_{15} c_{45}^{2}-d_{14} c_{55}^{2}\right) I_{3}\right.} \\
+ & d_{15} c_{44}^{2} I_{2} \\
& \left.-d_{45} c_{11}^{2} I_{1}\right] / 2 d_{15} c_{55}^{2} \\
& -2 d_{45} A(1,1,1,-1,-2) / c_{55}^{2}
\end{aligned}
$$




$$
\begin{aligned}
{[-1,1,1,-1,-4]=[} & \left(\beta_{5} / b_{5}-c_{45}^{2} / d_{45}-b_{1} c_{55}^{2} / b_{5} d_{15}\right) I_{3} \\
+ & c_{44}^{2} I_{2} / d_{45}+c_{11}^{2} I_{1} / d_{15} \\
& -4 A(1,1,1,-1,-2)] / 4 d_{15}
\end{aligned}
$$

\section{TABLE OF CUBIC CASES}

In addition to $(2.1)$ to $(2.5)$, the following definitions are used in cubic cases. They are obtained from (2.6) to (2.16), with omission of (2.10), by putting $a_{4}=1$ and $b_{4}=0$ and subsequently replacing $\left(a_{5}, b_{5}\right)$ by $\left(a_{4}, b_{4}\right)$ :

$$
\begin{aligned}
(x-y) M & =\left(X_{1}+Y_{1}\right)\left[(\xi+\eta)^{2}-h(x-y)^{2}\right]^{1 / 2} \\
& =\left(X_{1}+Y_{1}\right)[2 \xi \eta+2 f+g(x+y)+2 h x y]^{1 / 2},
\end{aligned}
$$

$$
\begin{gathered}
L_{ \pm}^{2}=M^{2}-\beta_{1} \pm(2 h)^{1 / 2} c_{11}, \quad W_{+}^{2}=M^{2}-b_{1}\left(c_{14}^{2}+c_{11} c_{44}\right) / d_{14} \\
(x-y) U=X_{1} \eta+Y_{1} \xi, \quad W^{2}=U^{2}-c_{11}^{2} b_{4} / 2 d_{14} \\
Q=X_{4} Y_{4} W / X_{1} Y_{1}, \quad P^{2}=Q^{2}+c_{44}^{2} b_{4} / 2 d_{14} \\
\rho=(2 h)^{1 / 2} c_{11}-\beta_{1}
\end{gathered}
$$

When one limit of integration is infinite, $M^{2}, U$, and $Q$ become, for $x=$ $+\infty$

$$
\begin{gathered}
M^{2}=b_{1}\left(2 h^{1 / 2} \eta+g+2 h y\right), \quad U=h^{1 / 2} Y_{1} \\
Q=\left(b_{4} / b_{1}\right)^{1 / 2} Y_{4} W / Y_{1},
\end{gathered}
$$

or, for $y=-\infty$,

$$
\begin{gathered}
M^{2}=-b_{1}\left(2 h^{1 / 2} \xi-g-2 h x\right), \quad U=h^{1 / 2} X_{1}, \\
Q=\left(b_{4} / b_{1}\right)^{1 / 2} X_{4} W / X_{1} .
\end{gathered}
$$

The three basic integrals (1.3) are

$$
I_{1 c}=4 R_{F}\left(M^{2}, L_{-}^{2}, L_{+}^{2}\right) \text {, }
$$

$$
\begin{gathered}
I_{2 c}=\left(2 c_{11}^{2} / 9 h\right)^{1 / 2}\left[4 \rho R_{D}\left(M^{2}, L_{-}^{2}, L_{+}^{2}\right)\right. \\
\left.-6 R_{F}\left(M^{2}, L_{-}^{2}, L_{+}^{2}\right)+3 / U\right]+2 X_{1} Y_{1} / U, \\
I_{3 c}=\left(2 c_{11} / 3 c_{44}\right)\left[\left(-4 b_{1} / d_{14}\right)\left(c_{14}^{2}+c_{11} c_{44}\right) R_{J}\left(M^{2}, L_{-}^{2}, L_{+}^{2}, W_{+}^{2}\right)\right. \\
\left.\quad-6 R_{F}\left(M^{2}, L_{-}^{2}, L_{+}^{2}\right)+3 R_{C}\left(U^{2}, W^{2}\right)\right] \\
+2 R_{C}\left(P^{2}, Q^{2}\right) .
\end{gathered}
$$

When one limit of integration is infinite, the integral (1.1) with $\prod b_{i} \neq 0$ converges only if $\sum p_{i}<-2$. To allow for the possibility of an infinite limit of 
integration, integrals with $\sum p_{i}<-2$ should not be reduced in terms of $I_{2 c}$, which has $\sum p_{i}=-1$ by (1.3). In [5] an integral called $J_{2 c}$ was used in such cases, but in the present context $J_{2 c}$ is complex and is replaced by

$$
\begin{aligned}
N_{2 c}=\left(8 h / 9 c_{11}^{2}\right)^{1 / 2}\left[4 \rho R_{D}\left(M^{2}, L_{-}^{2}, L_{+}^{2}\right)\right. & \\
& \left.-6 R_{F}\left(M^{2}, L_{-}^{2}, L_{+}^{2}\right)+3 / U\right]+2 / X_{1} Y_{1} U .
\end{aligned}
$$

The last term vanishes if one limit of integration is infinite.

Of the 40 cubic cases with $\sum\left|p_{i}\right| \leq 7$ and $\sum p_{i} \leq 3$ listed in [5], 20 have $p_{2}=p_{3}$. For brevity, some of the formulas contain the quantities $J_{1 c}$ and $K_{2 c}$, which can be computed in the present context from

$$
\begin{aligned}
J_{1 c} & =c_{11}^{2} I_{1 c} / 2-2 b_{1} A(1,1,1), \\
K_{2 c} & =c_{11}^{2} N_{2 c} / 2-2 d_{14} A(-1,1,1,-2) .
\end{aligned}
$$

In 18 of the 20 cases the formulas given in [5] can be used to evaluate

$$
\left[p_{1}, p_{2}, p_{2}, p_{4}\right]=\int_{y}^{x}\left(f+g t+h t^{2}\right)^{p_{2} / 2} \prod_{i=1,4}\left(a_{i}+b_{i} t\right)^{p_{i} / 2} d t
$$

by expressing coefficients involving $a_{2}, b_{2}, a_{3}, b_{3}$ in terms of $f, g$, and $h$ with the help of identities (2.19). Since the remaining two cases, as well as $[-3,-3,-3]$, involve the complex quantity $J_{2 c}$, they are listed here in terms of $N_{2 c}$ :

$$
\begin{gathered}
{[1,-3,-3]=\left[-c_{11}^{2} N_{2 c}-\beta_{1} I_{1 c}-2\left(\alpha_{1}+\beta_{1} x\right) / X_{1} \xi\right.} \\
\left.+2\left(\alpha_{1}+\beta_{1} y\right) / Y_{1} \eta\right] / \delta^{2}, \\
{[-1,-3,-3]=\left[\beta_{1} N_{2 c}+2 h I_{1 c}+2(g+2 h x) / X_{1} \xi\right.} \\
\left.-2(g+2 h y) / Y_{1} \eta\right] / \delta^{2}, \\
{[-3,-3,-3]=\left(2 / \delta^{2} c_{11}^{2}\right)\left[\left(2 b_{1}^{2} \delta^{2}-h c_{11}^{2}\right) N_{2 c}-h \beta_{1} I_{1 c}\right.} \\
\left.-2 h\left(\alpha_{1}+\beta_{1} x\right) / X_{1} \xi+2 h\left(\alpha_{1}+\beta_{1} y\right) / Y_{1} \eta\right] \\
+4 b_{1} A(-1,-1,-1) / c_{11}^{2} .
\end{gathered}
$$

Among integrals with $p_{2}=p_{3}, \sum\left|p_{i}\right| \leq 7$, and $\sum p_{i} \leq 3$, there are 12 that are not listed in [5] because the odd $p$ 's are not in descending order. Five are integrals of the second kind with $p_{4}=0$ :

$$
\begin{aligned}
{[-3,-1,-1] } & =N_{2 c}, \\
{[-1,1,1] } & =\left[\beta_{1} I_{2 c}+c_{11}^{2} I_{1 c}+2 b_{1} A(1,1,1)\right] / 3 b_{1}^{2}, \\
{[-3,1,1] } & =\left(2 h I_{2 c}+\beta_{1} I_{1 c}\right) / b_{1}^{2}-2 A(-1,1,1) / b_{1}, \\
{[-5,-1,-1] } & =\left(-2 / 3 c_{11}^{2}\right)\left[2 \beta_{1} N_{2 c}+h I_{1 c}+2 b_{1} A(-3,1,1)\right], \\
{[-5,1,1] } & =\left[\beta_{1} N_{2 c}+2 h I_{1 c}-2 b_{1} A(-3,1,1)\right] / 3 b_{1}^{2} .
\end{aligned}
$$


Three integrals of the second kind have $p_{4}=2$ with no restrictions on $a_{4}$ or $b_{4}$ :

$$
\begin{gathered}
{[-3,-1,-1,2]=\left(-d_{14} N_{2 c}+b_{4} I_{1 c}\right) / b_{1},} \\
{[-3,1,1,2]=\left[\left(b_{4} \beta_{1}-6 h d_{14}\right) I_{2 c}+\left(b_{4} c_{11}^{2}-3 d_{14} \beta_{1}\right) I_{1 c}\right] / 3 b_{1}^{3}} \\
+2\left[d_{14} A(-1,1,1)+b_{4} A(1,1,1) / 3\right] / b_{1}^{2} \\
{[-1,1,1,2]=\left[\left(2 b_{1}^{2} b_{4} \delta^{2}-h b_{4} c_{11}^{2}-5 h d_{14} \beta_{1}\right) I_{2 c}\right.} \\
\left.\quad-\left(b_{4} \beta_{1}+10 h d_{14}\right) J_{1 c}\right] / 15 h b_{1}^{3} \\
+2\left[b_{4} A(3,1,1) / 5-d_{14} A(1,1,1)\right] / b_{1}^{2} .
\end{gathered}
$$

The final four cases are integrals of the third kind with $p_{4}=-2$ or -4 :

$$
\begin{gathered}
{[-3,-1,-1,-2]=\left(b_{4}^{2} I_{3 c}-b_{1} d_{14} N_{2 c}-b_{1} b_{4} I_{1 c}\right) / d_{14}^{2},} \\
{[-3,1,1,-2]=\left[b_{1} c_{44}^{2} I_{3 c}-d_{14} c_{11}^{2} N_{2 c}\right.} \\
\left.-\left(b_{4} c_{11}^{2}+2 d_{14} \beta_{1}\right) I_{1 c}\right] / 2 b_{1} d_{14}^{2}, \\
{[-1,1,1-2]=\left(b_{1} c_{44}^{2} I_{3 c}+2 h d_{14} I_{2 c}-b_{4} c_{11}^{2} I_{1 c}\right) / 2 b_{1} b_{4} d_{14},} \\
{[-1,1,1,-4]=\left[\left(2 \beta_{4} d_{14}-b_{1} c_{44}^{2}\right) I_{3 c}+2 d_{14} K_{2 c}+b_{4} c_{11}^{2} I_{1 c}\right] / 4 b_{4} d_{14}^{2}}
\end{gathered}
$$

\section{THE BASIC INTEgrals}

To derive the expressions given in $\S \S 2$ and 3 for the seven integrals $I_{1}$, $I_{2}, \ldots, I_{3 c}$ in terms of real quantities, it suffices to deal with $I_{1}$ and $I_{3}$ because the other five can be obtained from these. Although $I_{1}$ was treated in [2], we shall redo it here to have uniform notation and to prepare for $I_{3}$.

By $[4,(2.13),(2.2),(2.3)]$ we have

$$
\begin{gathered}
I_{1}=[-1,-1,-1,-1]=2 R_{F}\left(U_{12}^{2}, U_{13}^{2}, U_{14}^{2}\right), \\
(x-y) U_{i j}=X_{i} X_{j} Y_{k} Y_{m}+Y_{i} Y_{j} X_{k} X_{m}, \\
X_{i}=\left(a_{i}+b_{i} x\right)^{1 / 2}, \quad Y_{i}=\left(a_{i}+b_{i} y\right)^{1 / 2},
\end{gathered}
$$

where $i, j, k, m$ is any permutation of $1,2,3,4$. Because $a_{1}+b_{1} t$ and $a_{4}+b_{4} t$ are assumed to be strictly positive on the open interval of integration, $X_{1}, Y_{1}, X_{4}$, and $Y_{4}$ are real and nonnegative. From $a_{2}=\bar{a}_{3}$ and $b_{2}=\bar{b}_{3}$, where an overbar denotes complex conjugation, it follows that $X_{2}=\bar{X}_{3}, Y_{2}=$ $\bar{Y}_{3}, U_{12}=\bar{U}_{13}$, and $U_{14} \geq 0$. The real quantities $f, g$, and $h$ satisfy

$$
\left(a_{2}+b_{2} t\right)\left(a_{3}+b_{3} t\right)=f+g t+h t^{2}>0, \quad-\infty<t<\infty .
$$


Because only $f, g$, and $h$ are given, we may choose $b_{2}=b_{3}=h^{1 / 2}$ and $\operatorname{Im}\left(a_{2}\right)>0$. If we assume $x$ and $y$ to be finite and take the principal branch of the square roots in (4.2), then $X_{2}$ and $Y_{2}$ lie in the open first quadrant of the complex plane, $X_{3}$ and $Y_{3}$ lie in the open fourth quadrant, and $X_{2} Y_{3}$ and $Y_{2} X_{3}$ have positive real part. Since we assume $x>y$ and since $X_{1} Y_{4}$ and $Y_{1} X_{4}$ cannot both vanish if $I_{1}$ is finite, we conclude that $\operatorname{Re} U_{12}=\operatorname{Re} U_{13}>0$.

The variables of $R_{F}$ can be made real and nonnegative by Landen's transformation $[6,(5.5)]$,

$$
\begin{gathered}
R_{F}\left(U_{12}^{2}, U_{13}^{2}, U_{14}^{2}\right)=2 R_{F}\left(M^{2}, L_{-}^{2}, L_{+}^{2}\right), \quad M=U_{12}+U_{13}, \\
L_{ \pm}=\left[\left(U_{12}+U_{14}\right)\left(U_{13}+U_{14}\right)\right]^{1 / 2} \pm\left[\left(U_{12}-U_{14}\right)\left(U_{13}-U_{14}\right)\right]^{1 / 2} \\
L_{+} L_{-}=2 M U_{14}, \quad L_{ \pm}^{2}-M^{2}=\left[\left(U_{14}^{2}-U_{12}^{2}\right)^{1 / 2} \pm\left(U_{14}^{2}-U_{13}^{2}\right)^{1 / 2}\right]^{2}
\end{gathered}
$$

Recalling that $U_{14} \geq 0, U_{12}=\bar{U}_{13}$, and $\operatorname{Re} U_{12}>0$, we see that $M>0$, $L_{+}>0$, and $L_{-} \geq 0$, with equality if and only if $U_{14}=0$ (the integral being then called complete). The last equation in (4.4) shows that $L_{+}^{2} \geq M^{2} \geq L_{-}^{2}$. Since

$$
(x-y) M=\left(X_{1} Y_{4}+Y_{1} X_{4}\right)\left(X_{2} Y_{3}+Y_{2} X_{3}\right)
$$

and

$$
\left(X_{2} Y_{3}+Y_{2} X_{3}\right)^{2}=\left(X_{2} X_{3}+Y_{2} Y_{3}\right)^{2}-\left(X_{2}^{2}-Y_{2}^{2}\right)\left(X_{3}^{2}-Y_{3}^{2}\right),
$$

we define

$$
\xi=X_{2} X_{3}=\left(f+g x+h x^{2}\right)^{1 / 2}, \quad \eta=Y_{2} Y_{3}=\left(f+g y+h y^{2}\right)^{1 / 2}
$$

and obtain

$$
(x-y) M=\left(X_{1} Y_{4}+Y_{1} X_{4}\right)\left[(\xi+\eta)^{2}-h(x-y)^{2}\right]^{1 / 2} .
$$

Equation (4.2) implies

$$
U_{i j}^{2}-U_{i k}^{2}=d_{i m} d_{j k}, \quad d_{i j}=a_{i} b_{j}-a_{j} b_{i},
$$

where $i, j, k, m$ is any permutation of $1,2,3,4$. From (4.4) we see that

$$
\begin{aligned}
L_{ \pm}^{2}-M^{2} & =\left[\left(d_{13} d_{42}\right)^{1 / 2} \pm\left(d_{12} d_{43}\right)^{1 / 2}\right]^{2} \\
& =d_{12} d_{43}+d_{42} d_{13} \pm\left(2 d_{12} d_{13}\right)^{1 / 2}\left(2 d_{42} d_{43}\right)^{1 / 2} \\
& =c_{14}^{2} \pm c_{11} c_{44},
\end{aligned}
$$

where

$$
\begin{aligned}
c_{i j}^{2} & =d_{i 2} d_{j 3}+d_{j 2} d_{i 3} \\
& =2 b_{2} b_{3} a_{i} a_{j}-\left(a_{2} b_{3}+a_{3} b_{2}\right)\left(a_{i} b_{j}+a_{j} b_{i}\right)+2 a_{2} a_{3} b_{i} b_{j} \\
& =2 f b_{i} b_{j}-g\left(a_{i} b_{j}+a_{j} b_{i}\right)+2 h a_{i} a_{j} .
\end{aligned}
$$

We note that the arguments of $R_{F}$ in (4.4) differ by amounts that are independent of $x$ and $y$. The assumption that $f+g t+h t^{2}>0$ for all real $t$ implies 
$f>0, h>0$, and $g^{2}-4 f h<0$. Hence, $c_{i i}^{2}$ is a positive definite quadratic form in the variables $a_{i}$ and $b_{i}$, and so we may take $c_{i i}$ to be real and positive.

The integral $I_{1}$ has now been expressed in terms of real quantities. Although the transformation (4.4) is unnecessary unless $f+g t+h t^{2}$ has complex zeros, it can still be used if $f+g t+h t^{2}$ has real zeros that do not interlace the zeros of $\left(a_{1}+b_{1} t\right)\left(a_{4}+b_{4} t\right)$. If they do interlace, the last member of the equation

$$
\left(L_{+}^{2}-L_{-}^{2}\right)^{2}=4 c_{11}^{2} c_{44}^{2}=16 d_{12} d_{13} d_{42} d_{43}
$$

is negative, and $L_{+}$and $L_{-}$are then conjugate complex.

We turn next to $I_{3}=[1,-1,-1,-1,-2]$, expressed by [4, (2.15), (2.5), (2.9)] as

$$
\begin{gathered}
I_{3}=\left(2 d_{12} d_{13} d_{14} / 3 d_{15}\right) R_{J}\left(U_{12}^{2}, U_{13}^{2}, U_{14}^{2}, W^{2}\right)+2 R_{C}\left(P^{2}, Q^{2}\right), \\
W^{2}=U_{14}^{2}-d_{12} d_{13} d_{45} / d_{15}, \quad Q=X_{5} Y_{5} W / X_{1} Y_{1}, \\
P^{2}=Q^{2}+d_{25} d_{35} d_{45} / d_{15} .
\end{gathered}
$$

Each $d$ or $U$ with a subscript 3 is the complex conjugate of the corresponding quantity with a subscript 2 . By (4.10) we have

$$
d_{12} d_{13}=c_{11}^{2} / 2, \quad d_{25} d_{35}=c_{55}^{2} / 2 .
$$

Since $U_{14} \geq 0, W^{2}$ and the arguments of $R_{C}$ are real, and we need only express $R_{J}$ in terms of real quantities by Landen's transformation. In [6, (8.5)] we put $(x, y, z, w)=\left(U_{12}, U_{13}, U_{14}, W\right)$ and $\left(\alpha, z_{ \pm}, w_{ \pm}\right)=\left(M, L_{ \pm}, W_{ \pm}\right) / 2$ to obtain

$$
\begin{aligned}
\frac{1}{4}\left(W_{+}^{2}-\right. & \left.W_{-}^{2}\right) R_{J}\left(U_{12}^{2}, U_{13}^{2}, U_{14}^{2}, W^{2}\right) \\
= & 4\left(W_{+}^{2}-M^{2}\right) R_{J}\left(M^{2}, L_{-}^{2}, L_{+}^{2}, W_{+}^{2}\right) \\
& -6 R_{F}\left(M^{2}, L_{-}^{2}, L_{+}^{2}\right)+3 R_{C}\left(U_{14}^{2}, W^{2}\right),
\end{aligned}
$$

where $M$ and $L_{ \pm}$are given by (4.4) and where [6, (7.2)] implies

$$
W_{ \pm}^{2}-M^{2}=\left[\left(W^{2}-U_{12}^{2}\right)^{1 / 2} \pm\left(W^{2}-U_{13}^{2}\right)^{1 / 2}\right]^{2} .
$$

From $[4,(2.9)]$ we find

$$
W^{2}-U_{12}^{2}=-d_{13} d_{14} d_{25} / d_{15}, \quad W^{2}-U_{13}^{2}=-d_{12} d_{14} d_{35} / d_{15},
$$

from which it follows by (4.10) that

$$
\begin{aligned}
W_{ \pm}^{2}-M^{2}= & \left(d_{14} / d_{15}\right)\left(-d_{13} d_{25}-d_{12} d_{35}\right) \\
& \pm 2\left(d_{14}^{2} / d_{15}^{2}\right)^{1 / 2}\left(d_{12} d_{13} d_{25} d_{35}\right)^{1 / 2} \\
= & \left(d_{14} / d_{15}\right)\left(c_{15}^{2} \pm c_{11} c_{55}\right) .
\end{aligned}
$$


We are free to choose $\left(d_{14}^{2} / d_{15}^{2}\right)^{1 / 2}$ to be $d_{14} / d_{15}$ regardless of the sign of the latter quantity because (4.14) still holds if $W_{+}$and $W_{-}$are interchanged (see the remark following $[6,(8.5)])$. Substitution in (4.14) and then in (4.12) leads to (2.15), wherein $U_{14}$ is abbreviated to $U$.

In $I_{3}$ we put $a_{5}=a_{4}$ and $b_{5}=b_{4}$ to get $I_{2}$, or $a_{5}=1$ and $b_{5}=0$ to get $I_{3}^{\prime}$. To obtain $I_{1 c}, I_{2 c}$, and $I_{3 c}$ from $I_{1}, I_{2}$, and $I_{3}$, we put $a_{4}=1$ and $b_{4}=0$ and subsequently replace the subscript 5 by 4 . The quantity $N_{2 c}$ is defined by

$$
c_{11}^{2} N_{2 c} / 2=h I_{2 c}-2 b_{1} A(-1,1,1) .
$$

Both terms on the right side become infinite if one limit of integration is infinite. Substitution of (3.9) and use of the identity

$$
h X_{1}^{2} Y_{1}^{2}-b_{1} U X_{1} Y_{1} A(-1,1,1)=c_{11}^{2} / 2
$$

lead to (3.11), in which all terms remain finite. We note from (3.17) that $N_{2 c}=[-3,-1,-1]$. From [5, (2.59)] we have

$$
K_{2 c}=h I_{2 c}-2 b_{4} A(1,1,1,-2) \text {, }
$$

which implies (3.12) by way of (4.18) and [4, (4.8)]. In deriving (3.14) to (3.16), it is necessary to use

$$
b_{3} J_{2 c}=h I_{2 c}-2 b_{3} A(1,1,-1)=c_{11}^{2} N_{2 c} / 2-2 d_{13} A(-1,1,-1),
$$

where the first equality comes from $[5,(2.17)]$ and the second from (4.18) and $[4,(4.8)]$.

Defining

$$
\delta^{2}=4 f h-g^{2}
$$

and noting from (4.3) that

$$
a_{2} b_{3}+a_{3} b_{2}=g, \quad\left(a_{2} b_{3}\right)\left(a_{3} b_{2}\right)=f h,
$$

we find

$$
2 a_{2} b_{3}=g+i \delta, \quad 2 a_{3} b_{2}=g-i \delta, \quad d_{23}=a_{2} b_{3}-a_{3} b_{2}=i \delta .
$$

The last equation is used in (2.19).

\section{USE OF RECURRENCE RELATIONS}

The 16 quartic cases $(2.23)$ to $(2.38)$ are obtained by recurrence relations with occasional help from the integrals listed in [4]. Let $e_{i}$ denote an $n$-tuple with 1 in the $i$ th place and 0 's elsewhere (for example, $\left[p+2 e_{1}\right]=\left[p_{1}+\right.$ $\left.\left.2, p_{2}, \ldots, p_{n}\right]\right)$. The four relations used most frequently are reproduced from [4] for convenience:

$$
\begin{gathered}
\left(p_{1}+\cdots+p_{n}+2\right) b_{i}[p]=\sum_{j \neq i} p_{j} d_{j i}\left[p-2 e_{j}\right]+2 A\left(p+2 e_{i}\right), \\
d_{i j}[p]=b_{j}\left[p+2 e_{i}\right]-b_{i}\left[p+2 e_{j}\right],
\end{gathered}
$$


$(\mathrm{C} i j)$

$$
\begin{gathered}
b_{j}[p]=b_{i}\left[p-2 e_{i}+2 e_{j}\right]+d_{i j}\left[p-2 e_{i}\right], \\
d_{i j}[p]=d_{k j}\left[p+2 e_{i}-2 e_{k}\right]+d_{i k}\left[p+2 e_{j}-2 e_{k}\right] .
\end{gathered}
$$

(Dijk)

A fifth relation $[3,(5.6)]$, with $i=1$ and $[p]=[-1,-1,-1,-3]$, is used to get (2.23). After obtaining $[-1,1,-1,-3]$ and $[-1,-1,1,-3]$ from (D142) and (D143), respectively, we can find $(2.24)$ by choosing $[p]=[-1,1,1,-3]$ in (A4), and we can find (2.25) from (D143). After obtaining [1, -1, -3, -1] from (D234) and interchanging subscripts 2 and 3 to get $[1,-3,-1,-1]$, we can find (2.26) from (B23) and also (2.27) from ( 42 ). Similarly, after obtaining $[-1,-1,-3,-1]$ from (B13) and interchanging subscripts 2 and 3 to get $[-1,-3,-1,-1]$, we can find (2.28) from (B23). The ten quartic cases of the third kind, (2.29) to (2.38), follow in order from (C42), (C31), (C41), (C54), (C45), (C15), (B15), (B45), (C45), and (B15).

To reduce the first cubic case, $(3.14)$, we first express $[1,-1,-3]$ in terms of $N_{2 c}$ by using [5, (2.25)] and (4.21), interchange $\left(a_{2}, b_{2}\right)$ with $\left(a_{3}, b_{3}\right)$ to get $[1,-3,-1]$, and then obtain $[1,-3,-3]$ from (B23). Exactly the same procedure, starting with $[-1,-1,-3]$ from $[5,(2.26)]$, yields (3.15). By choosing $[p]=[-1,-3,-3]$ and $i=1$ in $[3,(5.5)]$, we can then get (3.16). Equations (3.17), (3.18), and (3.19) follow from putting $a_{4}=1$ and $b_{4}=0$ in (2.23), (2.25), and (2.24), respectively. In the first case, and in later cases where necessary, $I_{2 c}$ is expressed in terms of $N_{2 c}$ by using (4.18). To get (3.20) we choose $[p]=[-3,-1,-1]$ and $i=1$ in $[3,(5.5)]$. The same procedure, with $[p]=[-3,1,1]$, yields $(3.21)$ with the help of the identity

$$
\begin{aligned}
b_{i} b_{j} A\left(p+4 e_{k}\right)+\left(b_{i} d_{j k}+b_{j} d_{i k}\right) A\left(p+2 e_{k}\right)+d_{i k} d_{j k} A(p) \\
\quad=b_{k}^{2} A\left(p+2 e_{i}+2 e_{j}\right) .
\end{aligned}
$$

Equations (3.22), (3.23), and (3.24) all come from (C41), while (3.25) and (3.26) come from (B14). The final two formulas, (3.27) and (3.28), are obtained by putting $a_{4}=1$ and $b_{4}=0$ and subsequently replacing the subscript 5 by 4 in (2.36) and (2.38), respectively.

\section{NUMERICAL CHECKS}

The 18 quartic cases in $\S 2$ and the 15 cubic cases in $\S 3$ were checked numerically when $x=2.0, y=0.5,\left(a_{1}, b_{1}\right)=(0.3,0.2),(f, g, h)=(0.4,-0.2$, $0.1),\left(a_{4}, b_{4}\right)=(0.9,-0.3)$, and $\left(a_{5}, b_{5}\right)=(0.4,0.5)$. In each case the integral on the left side, defined by (2.18) or (3.13), was integrated numerically by the SLATEC code QNG. On the right side the seven basic integrals $I_{1}$, $I_{2}, \ldots, I_{3 c}$ were calculated from (2.14) to (2.17) and (3.8) to (3.10) by using the codes for $R$-functions in the Supplements to $[3,4]$. The $A$ 's were calculated by a simple code, and the remaining calculations were done with a hand calculator. For each of the 33 cases the values obtained for the two sides agreed to better than one part in a million. 
Some intermediate values for the quartic cases are:

$$
\begin{aligned}
& M^{2}=0.62249271, \quad R_{F}\left(M^{2}, L_{-}^{2}, L_{+}^{2}\right)=1.2543726, \\
& L_{-}^{2}=0.54993185, \quad R_{D}\left(M^{2}, L_{-}^{2}, L_{+}^{2}\right)=1.7960842 \text {, } \\
& L_{+}^{2}=0.74305357, \quad R_{J}\left(M^{2}, L_{-}^{2}, L_{+}^{2}, W_{+}^{2}\right)=-0.99822609 \text {, } \\
& W_{+}^{2}=-0.54216139, \quad R_{C}\left(U^{2}, W^{2}\right)=1.7237432 \text {, } \\
& U^{2}=0.16410988, \quad R_{C}\left(P^{2}, Q^{2}\right)=0.98880184 \text {, } \\
& W^{2}=-0.13717583, \quad R_{J}\left(M^{2}, L_{-}^{2}, L_{+}^{2}, M^{2}+\rho\right)=1.5689637 \text {, } \\
& M^{2}+\rho=0.92172730, \quad R_{C}\left(U^{2}, W_{1}^{2}\right)=2.2358652 \text {, } \\
& W_{1}^{2}=0.21960988, \quad R_{C}\left(P_{1}^{2}, Q_{1}^{2}\right)=1.16864877 \text {, } \\
& \begin{array}{ll}
A(1,1,1,-1)=0.54975858, & I_{1}=5.0174903 \\
A(1,1,1,-1,-2)=0.04955294, & I_{2}=5.8882786 \\
A(-1,1,1,-1)=0.33929812, & I_{3}=2.7228427 \\
A(1,1,1,-3)=2.6651950, & I_{3}^{\prime}=2.7668674
\end{array}
\end{aligned}
$$

Some intermediate values for the cubic cases are:

$$
\begin{array}{lll}
M^{2}=1.1713435, & R_{F}\left(M^{2}, L_{-}^{2}, L_{+}^{2}\right)=0.89978529, \\
L_{-}^{2}=1.1496883, & R_{D}\left(M^{2}, L_{-}^{2}, L_{+}^{2}\right)=0.67751039, \\
L_{+}^{2}=1.3929988, & R_{J}\left(M^{2}, L_{-}^{2}, L_{+}^{2}, W_{+}^{2}\right)=0.71986645, \\
W_{+}^{2}=1.2606479, & R_{C}\left(U^{2}, W^{2}\right)=1.7844272, \\
U^{2}=0.34181141, & R_{C}\left(P^{2}, Q^{2}\right)=1.9470611, \\
W^{2}=0.30070030, & & \\
A(-1,-1,-1)=-0.88367862, & I_{1 c}=3.5991412, \\
A(-1,1,1)=-0.14545887, & I_{2 c}=1.9453098, \\
A(-1,1,1,-2)=1.3179127, & I_{3 c}=4.0022901, \\
A(1,1,1)=0.16859514, & N_{2 c}=6.8301223, \\
A(-3,1,1)=-1.1735711, & J_{1 c}=0.06573017, \\
A(3,1,1)=0.22618313, & K_{1 c}=0.96438740 .
\end{array}
$$

\section{BIBLIOGRAPHY}

1. P. F. Byrd and M. D. Friedman, Handbook of elliptic integrals for engineers and scientists, 2nd ed., Springer-Verlag, New York, 1971.

2. B. C. Carlson, Elliptic integrals of the first kind, SIAM J. Math. Anal. 8 (1977), 231-242.

3. __ A table of elliptic integrals of the second kind, Math. Comp. 49 (1987), 595-606. (Supplement, ibid., S13-S17.)

4. _ A table of elliptic integrals of the third kind, Math Comp. 51 (1988), 267-280. (Supplement, ibid., S1-S5.)

5. __ _ A table of elliptic integrals: cubic cases, Math. Comp. 53 (1989), 327-333.

6. __ Landen transformations of integrals, Asymptotic and Computational Analysis ( $R$. Wong, ed.), Marcel Dekker, New York, 1990. 
7. I. S. Gradshteyn and I. M. Ryzhik, Table of integrals, series, and products, Academic Press, New York, 1980.

8. A. P. Prudnikov, Yu. A. Brychkov, and O. I. Marichev, Integrals and series, Vol. 1, Gordon and Breach, New York, 1986.

Ames laboratory and Department of Mathematics, Iowa State University, Ames, IowA 50011

E-mail address: s1.mth@isumvs.bitnet 